ARTICLE

https://doi.org/10.1038/s41467-019-10612-3

\title{
Unveiling the synergistic effect of precursor stoichiometry and interfacial reactions for perovskite light-emitting diodes
}

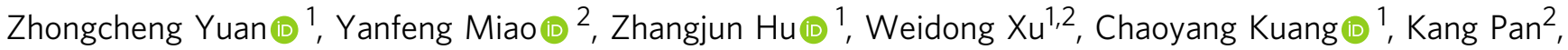

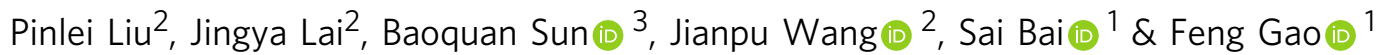

Metal halide perovskites are emerging as promising semiconductors for cost-effective and high-performance light-emitting diodes (LEDs). Previous investigations have focused on the optimisation of the emissive perovskite layer, for example, through quantum confinement to enhance the radiative recombination or through defect passivation to decrease non-radiative recombination. However, an in-depth understanding of how the buried charge transport layers affect the perovskite crystallisation, though of critical importance, is currently missing for perovskite LEDs. Here, we reveal synergistic effect of precursor stoichiometry and interfacial reactions for perovskite LEDs, and establish useful guidelines for rational device optimization. We reveal that efficient deprotonation of the undesirable organic cations by a metal oxide interlayer with a high isoelectric point is critical to promote the transition of intermediate phases to highly emissive perovskite films. Combining our findings with effective defect passivation of the active layer, we achieve high-efficiency perovskite LEDs with a maximum external quantum efficiency of $19.6 \%$.

\footnotetext{
${ }^{1}$ Department of Physics, Chemistry and Biology (IFM), Linköping University, Linköping SE-58183, Sweden. ${ }^{2}$ Key Laboratory of Flexible Electronics (KLOFE) and Institute of Advanced Materials (IAM), Jiangsu National Synergetic Innovation Center for Advanced Materials (SICAM), Nanjing Tech University (Nanjing Tech), Nanjing 211816, China. ${ }^{3}$ Jiangsu Key Laboratory for Carbon-Based Functional Materials \& Devices, Institute of Functional Nano \& Soft Materials (FUNSOM), Joint International Research Laboratory of Carbon-Based Functional Materials and Devices, Soochow University, Suzhou 215123, China. Correspondence and requests for materials should be addressed to S.B. (email: sai.bai@liu.se) or to F.G. (email: feng.gao@liu.se)
} 
S olution-processed metal halide perovskites have demonstrated great advances in the applications of highperformance light-emitting diodes (LEDs), due to their high photoluminescence (PL) efficiencies, pure and widely tuneable light emission, and excellent charge transport properties ${ }^{1-5}$. In a typical perovskite LED, the luminescent perovskite film is sandwiched between the electron- and hole-injection interlayers with suitable energy levels and electrical properties ${ }^{6-8}$. Both the perovskite active layer and charge-injection layers are of critical importance to achieve high-efficiency perovskite $\operatorname{LEDs}^{3,9,10}$. Previous studies have focused on the optimisation of the emissive layer through solution engineering of the perovskite precursors, such as tuning the perovskite dimension, manipulating the precursor composition, incorporating additives or passivation molecules ${ }^{6,11-14}$. However, experience from the development of perovskite solar cells indicates that the surface properties of underlying interlayers also significantly affect the perovskite active layer deposited on top ${ }^{15,16}$. The surface chemical and physical properties of the buried interlayers can affect the perovskite crystallization process, which in turn influences the film quality of the perovskite active layer and consequently the ensuing device performance ${ }^{17,18}$. In addition, the possible reactions between the interlayers and the perovskites even add more complexity ${ }^{19}$. Despite rapid improvements in the external quantum efficiencies (EQEs) of perovskite LEDs during the past few years $1,6,20-22$, an in-depth understanding of the combined effects of the perovskite precursor stoichiometry and the interfacial properties on the resulting perovskite films and devices is yet currently lacking.

Here we investigate perovskite films and LEDs fabricated on commonly used n-type metal oxide electron-transporting layers (ETLs), e.g., zinc oxide $(\mathrm{ZnO})$, titanium oxide $\left(\mathrm{TiO}_{x}\right)$ and tin oxide $\left(\mathrm{SnO}_{2}\right)$, modified with an ultra-thin polyethylenimine ethoxylated (PEIE) layer. We demonstrate that incorporating excess organic halides in the precursor greatly suppresses the non-radiative recombination in the resulting films, leading to highly emissive perovskite active layers and efficient LEDs. The deprotonation of excess organic cations by the basic $\mathrm{ZnO}$ surface is critical to trigger the transition of precursor intermediates into high-quality perovskite light emitters. We demonstrate that this interface-induced deprotonation process is compatible with further defect passivation strategies. We passivate the perovskite layers with an efficient diamine molecule, 4,7,10-trioxa-1,13-tridecanediamine (TTDDA), and obtain high-efficiency near-infrared (NIR) LEDs with a maximum EQE of $19.6 \%$ based on formamidinium (FA)-cesium (Cs) mixed-cation perovskites.

\section{Results}

Impacts of perovskite precursor stoichiometry. We show the device geometry of our perovskite LEDs in Fig. 1a. ZnO/PEIE and poly $[(9,9-$ dioctylfluorenyl-2,7-diyl)-co-(4,4'-(N-(4-secbutylphenyl) diphenylamine)) (TFB) are used as the electronand hole-injection layers, respectively ${ }^{1,6}$. We employ a 'thermally stable' formamidinium lead triode $\left(\mathrm{FAPbI}_{3}\right)$ perovskite as the light-emitting material, with $10 \%$ of formamidinium iodide (FAI) in the precursor substituted by cesium iodide (CsI) to push the Goldschmidt tolerance factor to a more stable region $^{23}$. We tune the molar ratio $(x)$ between $(0.9 \mathrm{FAI}+$ $0.1 \mathrm{CsI})$ and lead iodide $\left(\mathrm{PbI}_{2}\right)(x=1.0,1.5,2.0,2.5$ and 3.0) to evaluate the effects of the precursor stoichiometry on the resulting films and devices. In order to focus our investigation on the precursor stoichiometry on its own, no additives or passivation molecules are added in the precursor solutions at this stage. All the perovskite active layers are deposited from a simple one-step spin-coating process and annealed in a glovebox at $100^{\circ} \mathrm{C}$ for $10 \mathrm{~min}$ (experimental details can be found in the "Methods" section).

We show the impact of increasing amount of organic halides on the device performance in Fig. 1b-d and summarize the device parameters in Supplementary Table 1. We are not able to obtain emitting devices from precursor with the stoichiometric ratio $(x=1.0)$ due to the formation of non-perovskite $\delta$-phase in the film (Supplementary Figure 1a), which shows almost no PL emission (Supplementary Figure 1b). The result is consistent with previous reports that a high temperature above $100{ }^{\circ} \mathrm{C}$ is generally required to obtain pure $\alpha$-phase FA-Cs mixed-cation iodide perovskites when processed from the stoichiometric precursor without the use of additives or antisolvents ${ }^{23-25}$. We show current density-radiance and voltage $(J \& R-V)$, EQE-current density (EQE- J) curves, and histograms of the peak EQEs of the devices prepared from precursors with different $x$ values in Fig. 1b-d. We observe enhanced EQEs and radiances with increasing $x$ values from 1.5 to 2.5 . For the champion device with $x=2.5$, we obtain a maximum EQE of $13.1 \%$ and a peak radiance of $206.6 \mathrm{~W} \mathrm{sr}^{-1} \mathrm{~m}^{-2}$. However, a further increase of $x$ to 3.0 results in a declined EQE of $6.4 \%$, along with much lower radiance of $13.6 \mathrm{~W} \mathrm{sr}^{-1} \mathrm{~m}^{-2}$ and obvious efficiency roll-off. We notice that the current density from the $x=3.0$ device is much lower than those processed from precursors with smaller $x$ values (Fig. 1c). The existence of a large amount of excess organic halides in this case is likely to affect the electronic properties of the resulting perovskites and introduce barriers for charge injection into the films, resulting in the decreased EQE and obvious efficiency roll-off ${ }^{26}$.

We perform a range of the film characterizations to understand the effects of excess organic components on the film quality and the ensuing device performance. As the X-ray diffraction (XRD) patterns of the perovskite films show in Fig. 2a, we observe a clear evolution of the perovskite crystal structures from a mixture of $\alpha /$ $\delta$-phase to pure $\alpha$-phase with increasing $x$ values from 1.5 to 3.0. The result is consistent with a previous study showing that excess FAI in the precursor is helpful for the formation of $\alpha$-phase perovskite at a low processing temperature ${ }^{27}$.

The films with higher $x$ values show much enhanced PL intensity, suggesting that excess organic halides effectively passivate the defects in the resulting films (Supplementary Figure 2a). According to the light-intensity-dependent PL quantum efficiency (PLQE) results in Fig. 2b, we observe suppressed non-radiative recombination in perovskite films processed with increasing organic halides. High PLQEs of over $50 \%$ are obtained for films $(x=3.0$ and 2.5$)$ under excitation of different light intensities from 0.15 to $300 \mathrm{~mW} \mathrm{~cm}^{-2}$. In contrast, the PLQEs of perovskite films processed from precursors with smaller $x$ values $(x=1.5$ and 2.0) are much lower under low excitation intensities and keep increasing with increasing excitation intensities, indicating a trap filling process due to the existence of defects in the perovskite films ${ }^{28}$. We further conduct time-correlated single photon counting (TCSPC) measurements to investigate the charge carrier decay dynamics (Fig. 2c). Consistent with the PLQE results, the PL lifetime increases with the $x$ value increasing from 1.5 to 2.5 . Considering the further improved PLQE value in the $x=3.0$ sample, the slight decrease of the PL lifetime in this case is ascribed to accelerated radiative recombination ${ }^{13}$, rather than the existence of more defects.

Different precursor stoichiometry also results in morphology changes in the obtained perovskite films. From the scanning electron microscopy (SEM) measurements (Fig. 2d), we observe reduced film coverage for perovskite films prepared from precursors with increasing $x$ values. This non-continuous perovskite film morphology was demonstrated beneficial to 
a

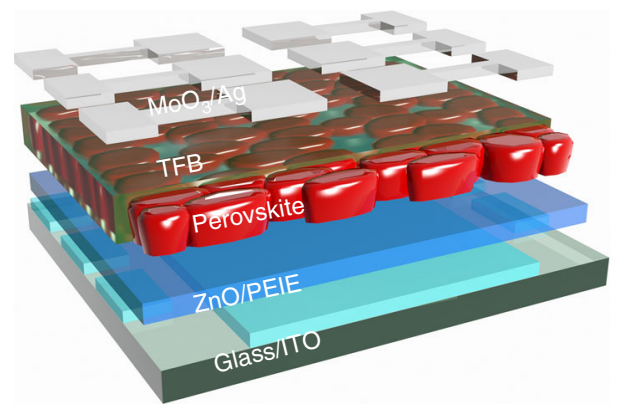

C

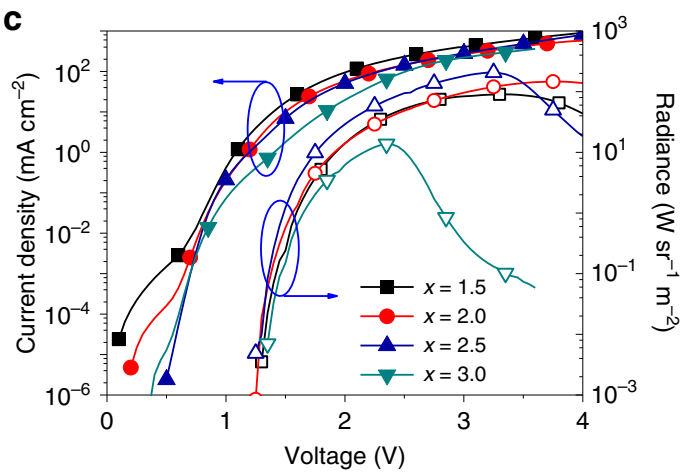

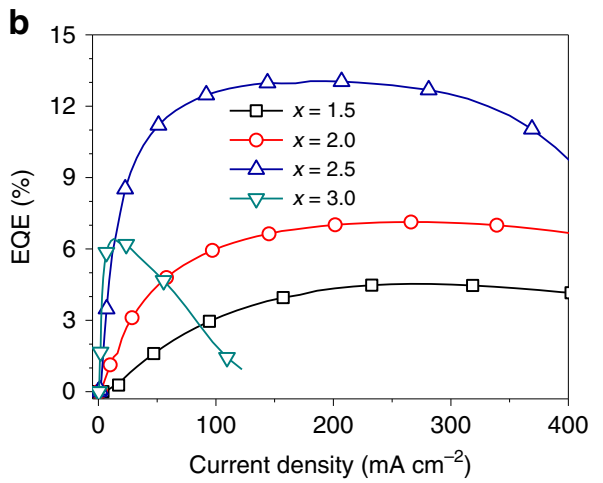

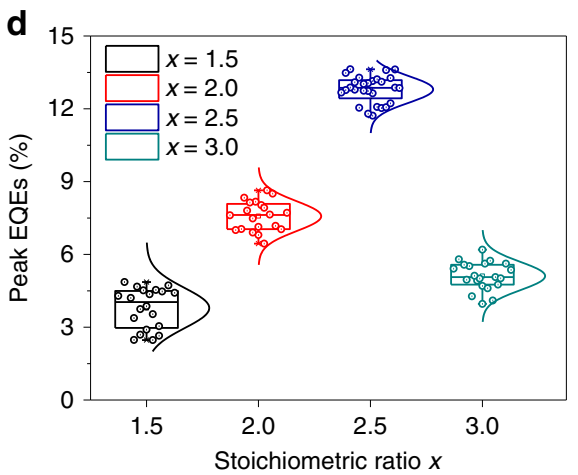

Fig. 1 Perovskite LEDs processed from precursors with excess FAI. a Schematic diagram of the device architecture of the perovskite LEDs in this work (ITO/ZnO/PEIE/perovskite/TFB/ $\mathrm{MoO}_{3} / \mathrm{Ag}$ ). b-d EQE-J (b), $J \& R$ - $V$ curves (c), and statistical distributions of peak EQEs (d) of perovskite LEDs fabricated from precursors with different $x$ values $(x=1.5,2.0,2.5$ and 3.0). For all box plots, the line in the center is the median value. The interquartile range (IQR) represents 25th-75th percentile of the EQE values. Whiskers show the extent of the whole EQE values, extending to 1.5 IQR in box-plots

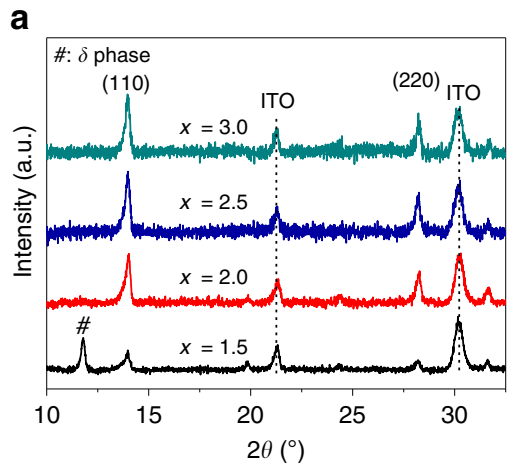

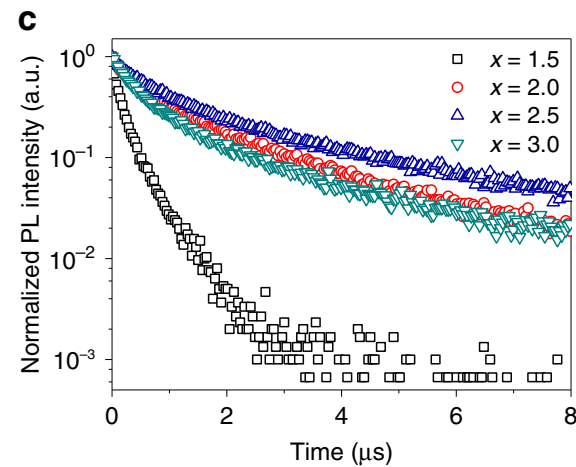

d
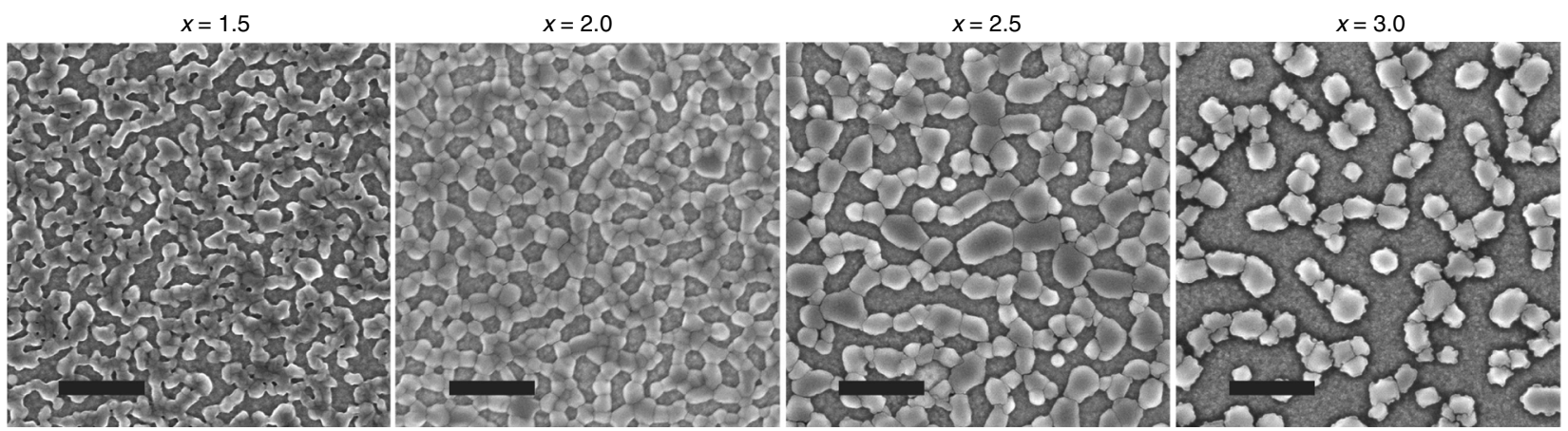

Fig. 2 Perovskite films processed from precursors with different component ratios. a-d XRD patterns (a), light-intensity-dependent PLQEs (b), PL decays (c) and SEM images (d) of thermal annealed perovskite films processed from precursors with different $x$ values $(x=1.5,2.0,2.5$ and 3.0). The scale bars in the SEM images are $1 \mu \mathrm{m}$ 

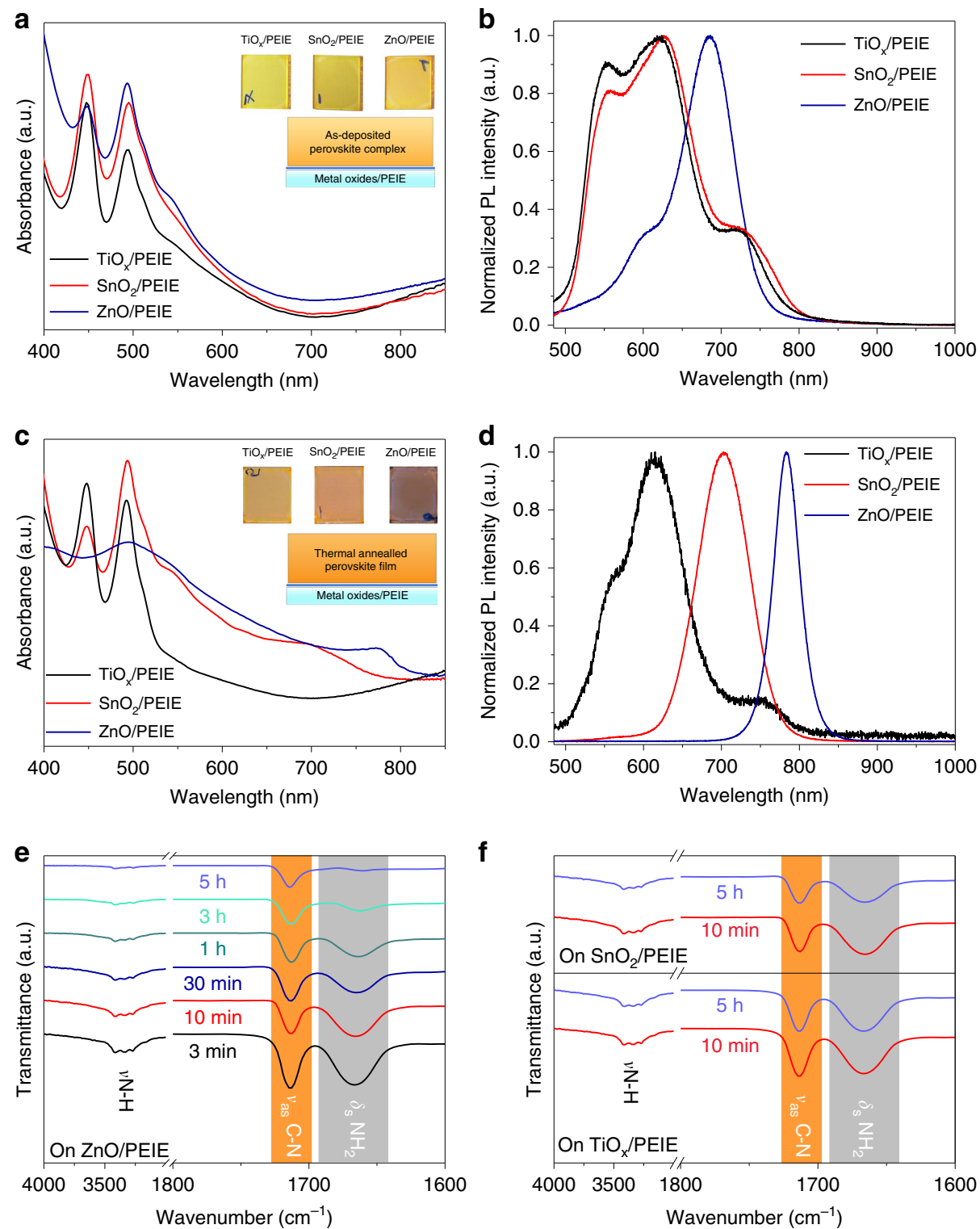

Fig. 3 Optical characteristics of perovskite films $(x=2.5)$ on different substrates. $\mathbf{a}-\mathbf{d}$ UV-Vis absorption $(\mathbf{a}, \mathbf{c})$ and normalized PL spectra $(\mathbf{b}, \mathbf{d})$ of the perovskite films on $\mathrm{TiO}_{x} / \mathrm{PEIE}, \mathrm{SnO}_{2} / \mathrm{PEIE}$ and $\mathrm{ZnO} / \mathrm{PEIE}$ substrates, respectively before $(\mathbf{a}, \mathbf{b})$ and after the thermal annealing (c, $\left.\mathbf{d}\right)$. Insets show the corresponding photographs of the perovskite films on different substrates. e-f FTIR spectra of perovskite films on $\mathrm{ZnO} / \mathrm{PEIE}(\mathbf{e}), \mathrm{TiO}_{x} / \mathrm{PEIE}$ and $\mathrm{SnO} 2 / \mathrm{PEIE}$ (f) substrates with different annealing time

improve the light-outcoupling efficiency ${ }^{1}$. However, the perovskite film processed from the $x=3.0$ precursor shows dramatically low film coverage, which is possibly one of the reasons for the strong efficiency roll-off under high current densities in the resulting perovskite LEDs.

Effects of the buried interface on the perovskite films. Having demonstrated the importance of the precursor stoichiometry for the fabrication of high-efficiency perovskite LEDs, however, we cannot obtain working devices when we replace the $\mathrm{ZnO}$ with two other n-type metal oxide thin films that are commonly used in perovskite optoelectronic devices, i.e., $\mathrm{TiO}_{x}$ and $\mathrm{SnO}_{2}{ }^{29,30}$.

We now proceed to understand the reasons why different metal oxides result in significant difference in the device performance. Considering similar electronic properties of the three metal oxides and the same hole injection contact on top, we focus on the perovskite layers on different metal oxides. We first characterize the as-deposited precursor films $(x=2.5)$ on different substrates (before the thermal annealing), and observe two strong exciton absorption peaks at around $450 \mathrm{~nm}$ and $500 \mathrm{~nm}$ from the ultraviolet-visible (UV-Vis) absorption spectra (Fig. 3a). The result is consistent with previously demonstrated ground-state excitations from the low-dimensional coordinated intermediate complexes containing excess methylammonium iodide (MAI), e.g., one-dimensional (1D) perovskite chains or self-assembled two-dimensional (2D) perovskite sheets in the asdeposited precursor films ${ }^{31,32}$.

We believe that the formation of these intermediate complexes is caused by the introduced excess organic halides, which hinder the sharing of iodides between different lead polyiodides octahedral, resulting in colloids with decreased sizes in the perovskite precursors. The different colloid sizes in the precursors with different $x$ values are confirmed by the dynamic light scattering (DLS) results (Supplementary Figure 3), which show a decreasing trend from around $163.0 \mathrm{~nm}(x=1.0)$ to around 
$3.6 \mathrm{~nm}(x=3.0)$. We also note that different substrates already result in difference in these intermediate complexes. For the perovskite on the $\mathrm{ZnO} / \mathrm{PEIE}$ substrate, we observe an additional absorption shoulder locating at around $550 \mathrm{~nm}$, which may origin from the soft-packing or self-assembly of the intermediate phases in the complex films ${ }^{31}$. The emergence of this absorption shoulder indicates aggregation of the intermediate phases, consistent with the obvious red-shift in the PL emission (Fig. 3b). These results indicate that the perovskite precursor complexes on the $\mathrm{ZnO} / \mathrm{PEIE}$ show more obvious aggregation right after the spin-coating or during the film drying process, which is different from those on the other two substrates.

After thermal annealing $(x=2.5)$, we observe negligible difference in the main diffraction peaks of the XRD results of the three samples, all showing pure $\alpha$-phase perovskite features (Supplementary Figure 4$)^{27}$. The perovskite film on $\mathrm{ZnO} / \mathrm{PEIE}$ shows enhanced diffraction intensity, suggesting improved crystallinity of the film. Despite similar XRD results, we observe significant difference in the absorption and emission spectra of the perovskite films on different substrates (Fig. 3c, d). We observe typical absorption and PL spectra of the $\alpha$-phase perovskites from the films on $\mathrm{ZnO} / \mathrm{PEIE}$. In contrast, both the absorption and PL spectra of the films on $\mathrm{TiO}_{\mathrm{x}} / \mathrm{PEIE}$ show negligible changes after thermal annealing, as compared with those of the intermediate phases in the as-deposited precursor complexes. For perovskites on $\mathrm{SnO}_{2} / \mathrm{PEIE}$, although we observe redshift of the absorption and PL spectra as compared with the precursor complexes, both the absorption and emission peaks locate at higher energies compared with the film on the $\mathrm{ZnO} /$ PEIE substrate. Difference in films on the three substrates is also clearly visible from the optical images of the resulting perovskite films (insets of Fig. 3a, c). The result suggests that the films deposited on $\mathrm{SnO}_{2} / \mathrm{PEIE}$ and $\mathrm{TiO}_{\mathrm{x}} / \mathrm{PEIE}$ mostly retain their intermediate phases, which are in high disorder. These intermediate phases show no obvious diffraction peaks in the XRD patterns, but are discernible from the optical characterizations. We conclude that, under the relatively low processing temperature of $100^{\circ} \mathrm{C}$ we use here, the efficient transition from precursor complexes to pure-phase and highly luminescent perovskite crystals only happens on the $\mathrm{ZnO} / \mathrm{PEIE}$ substrate.
Role of $\mathrm{FA}^{+}$deprotonation for perovskite crystallization. The different electrical, structural and optical properties of the resulting perovskite films suggest that the perovskite crystallization is highly related to the buried substrates. We notice that the three n-type metal oxides exhibit different surface chemical properties. The measured isoelectric point (IEP) values, which represent the surface acidity of the metal oxide films, are reported to be 3.5-6.2 for $\mathrm{TiO}_{x}$ (acidic), 6.6-9.5 for $\mathrm{SnO}_{2}$ (neutral) and 8.7-10.3 for $\mathrm{ZnO}$ (basic), respectively ${ }^{33}$. As demonstrated by previous theoretical calculations and experimental results, the organic cations may experience a deprotonation process upon in contact with metal oxide films. The deprotonation probability depends on the surface chemical properties of the metal oxide, of which a basic surface with a higher IEP value is likely to induce an easier deprotonation of the organic cations ${ }^{19,34}$. We anticipate that the ultra-thin PEIE layer used here cannot completely prevent the direct contact and interactions between the perovskite complexes and metal oxides. This is also consistent with the observed absorption shoulder at around $550 \mathrm{~nm}$ and the redshifted PL spectrum of the as-deposited precursor complex on $\mathrm{ZnO} / \mathrm{PEIE}$, resulting from the interaction between perovskites and $\mathrm{ZnO}$ (Fig. 3a, b).

To further explore the difference of substrates-induced deprotonation of the organic cations, we conduct the Fourier transform infrared (FTIR) spectroscopy measurements to monitor the evolution of the FAI molecules during the perovskite crystallization. We first collect the FTIR spectra of the pristine FAI and the perovskite films processed from precursor solutions with different $x$ values on $\mathrm{ZnO} / \mathrm{PEIE}$. In the pristine FAI film, the peaks locating at $3420-3260,1714$ and $1666 \mathrm{~cm}^{-1}$ can be ascribed to $\mathrm{N}-\mathrm{H}$ stretching $(v \mathrm{~N}-\mathrm{H}), \mathrm{C}-\mathrm{N}$ stretching $\left(v_{\mathrm{as}} \mathrm{C}-\mathrm{N}\right)$ and $-\mathrm{NH}_{2}$ scissoring vibrations $\left(v_{\mathrm{s}} \mathrm{NH}_{2}\right)$, respectively (Supplementary Figure $5 \mathrm{a})^{35}$. In the perovskite film processed from the stoichiometric precursor $(x=1.0)$, the $v_{\mathrm{s}} \mathrm{NH}_{2}$ is greatly restricted because of the formation of $\mathrm{N}-\mathrm{H} \cdots \mathrm{I}$ hydrogen bonds within the crystal structure ${ }^{36,37}$. The $v_{\mathrm{s}} \mathrm{NH}_{2}$ becomes more obvious under organic cation-rich conditions $(x=2.0$ and $x=3.0)$ due to the existence of FAI molecules in the adjacent area outside of the perovskite crystals (Supplementary Figure $5 \mathrm{~b})^{1,38}$. Therefore, the relative peak intensity of $v_{\mathrm{s}} \mathrm{NH}_{2}$ at $1666 \mathrm{~cm}^{-1}$ can be used to
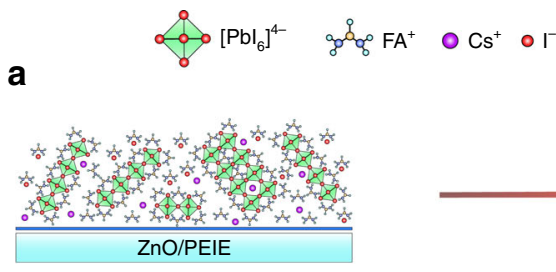

b
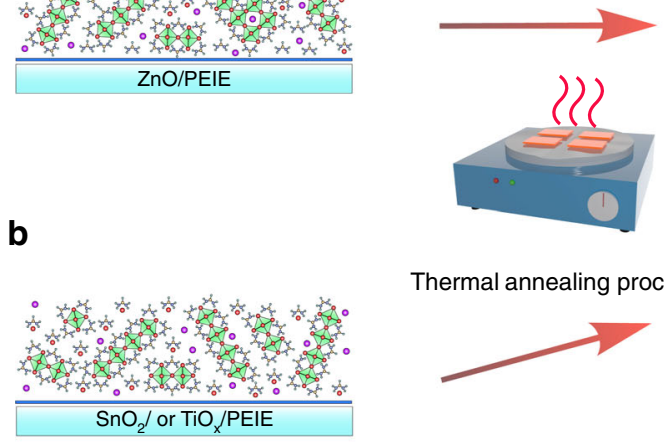

Thermal annealing process

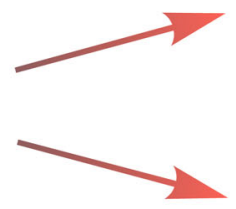

As-deposited precursor complex $(x=2.5)$
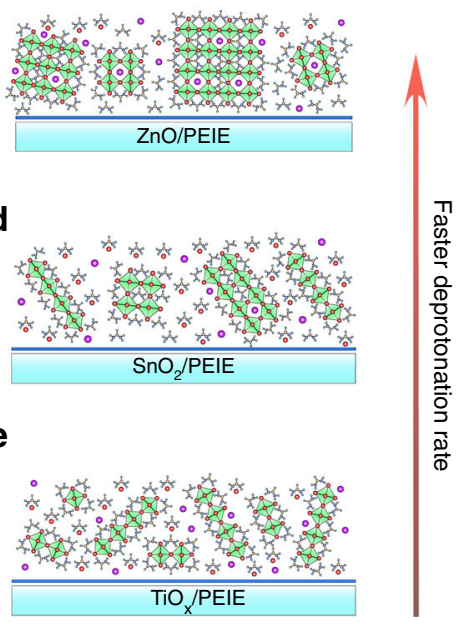

Thermal annealled perovskite film

Fig. 4 Schematic illustration of perovskite $(x=2.5)$ crystallization on different substrates. $\mathbf{a}, \mathbf{b}$ The as deposited perovskite precursor complexes on ZnO/ PEIE (a) and on $\mathrm{TiO}_{x} / \mathrm{PEIE}$ or $\mathrm{SnO}_{2} / \mathrm{PEIE}$ (b) substrates. c-e The thermal annealed perovskite films on $\mathrm{ZnO} / \mathrm{PEIE}(\mathbf{c}), \mathrm{SnO}_{2} / \mathrm{PEIE}(\mathbf{d})$ and TiO $x / \mathrm{PEIE}(\mathbf{e})$ substrates, respectively 
monitor the amount of excess FAI molecules within the films. As shown in Fig. 3e, we observe a continuous decrease in the intensity of $v \mathrm{~N}-\mathrm{H}$ and $v_{\text {as }} \mathrm{C}-\mathrm{N}$ of FAI molecules on $\mathrm{ZnO} / \mathrm{PEIE}$, indicating a continuous deprotonation of the excess $\mathrm{FA}^{+}$cations in the perovskite films during the thermal annealing ${ }^{35}$. Meanwhile, the $v_{\mathrm{s}} \mathrm{NH}_{2}$ at $1666 \mathrm{~cm}^{-1}$ shows a faster decline as compared with that of $v_{\text {as }} \mathrm{C}-\mathrm{N}$, suggesting that the $\mathrm{FA}^{+}$cations outside the crystal lattice experience a faster deprotonation rate during the film crystallization (Supplementary Figure 6a). On the contrary, the FTIR results of the perovskite films show almost no change on $\mathrm{TiO}_{\mathrm{x}} / \mathrm{PEIE}$ or a slight decrease on $\mathrm{SnO}_{2} / \mathrm{PEIE}$ substrate after $5 \mathrm{~h}$ thermal annealing (Fig. $3 \mathrm{f}$ and Supplementary Figure 6b). We observe slight interfacial reactions of the organic cations on $\mathrm{SnO}_{2} /$ PEIE due to a higher IEP value of $\mathrm{SnO}_{2}$ compared to that of $\mathrm{TiO}_{x}$, suggesting that the recently observed thermal instability of perovskite solar cells based on bare $\mathrm{SnO}_{2}$ is also possibly related to the potential reaction at the perovskite $/ \mathrm{SnO}_{2}$ interface ${ }^{39}$. These FTIR results are consistent with the reported IEP values of the metal oxides and further demonstrate the easier deprotonation of $\mathrm{FA}^{+}$cations on basic metal oxide substrates, e.g., $\mathrm{ZnO}$ in our case.

We now obtain a clear picture of the crystallization process and film properties of the perovskite films on the three substrates. As we show in Fig. 4, in the as-deposited precursor complexes (organic components-rich condition, e.g., $x=2.5$ ), most of the excess FAI exist as molecules in the form of ionic pairs with strong hydrogen bonds between $\mathrm{N}-\mathrm{H} \cdots \mathrm{N}$. The corner sharing $\left[\mathrm{PbI}_{6}\right]^{4-}$ units are surrounded by the abundant organic halides, which inhibit the formation of 3-Dimentional (3D) perovskite phases, easily generating low-dimensional intermediate phases on the substrates (Fig. $4 \mathrm{a}, \mathrm{b}$ ). Considering the high decomposition temperature of FAI $\left(246^{\circ} \mathrm{C}\right)$, the mild thermal annealing condition $\left(100^{\circ} \mathrm{C}\right)$ we used here is not able to decompose and eliminate the abundant FAI molecules on the
$\mathrm{SnO}_{2} / \mathrm{PEIE}$ and $\mathrm{TiO}_{\mathrm{x}} / \mathrm{PEIE}$ substrates. This consequently hinders the transition of intermediate phases to high-quality perovskite crystals during the thermal annealing process (Fig. $4 \mathrm{~d}, \mathrm{e})^{36}$. In contrast, the basic $\mathrm{ZnO} / \mathrm{PEIE}$ interface induces efficient deprotonation of the organic cations, providing external energy to remove the FAI molecules during the thermal annealing. We note that, even after prolonged thermal annealing process $\left(100^{\circ} \mathrm{C}\right.$ for up to $5 \mathrm{~h}$ ), the films on $\mathrm{SnO}_{2}$ / PEIE and $\mathrm{TiO}_{\mathrm{x}} / \mathrm{PEIE}$ substrates remain at their intermediate states as evidenced by the photographs, UV-vis absorption and PL spectra (Supplementary Figure $7 \mathrm{a}-\mathrm{c}$ ), apart from slightly enhanced crystallinity according to the XRD patterns (Supplementary Figure 7d). We anticipate that the $\mathrm{ZnO}$-induced efficient deprotonation of the organic cations removes the crystallization barriers, reduces the activation energy for perovskite crystallization, and hence promotes the phase transition from the intermediate phases to $\alpha$-phase perovskite crystals, generating high-quality emissive perovskite active layers (Fig. 4c).

We note that, the thermal annealing of perovskite films on $\mathrm{ZnO} / \mathrm{PEIE}$ substrates needs to be carefully controlled to obtain optimized crystalline emitters without further decomposing the as-formed perovskite active layer. From the XRD patterns of perovskite films with different annealing time (Supplementary Figure 8), we observe no $\mathrm{PbI}_{2}\left(11.8^{\circ}\right)$ from the films with $30 \mathrm{~min}$ annealing. However, further extending the annealing time to $1 \mathrm{~h}$ results in the formation of obvious $\mathrm{PbI}_{2}$ in the film ${ }^{19}$. These results indicate that at the beginning of the thermal annealing, the deprotonation process by $\mathrm{ZnO} / \mathrm{PEIE}$ substrates mainly contributes to phase transition rather than the perovskite decomposition. However, a long-term annealing of the perovskite film on $\mathrm{ZnO} / \mathrm{PEIE}$ causes decomposition of the as-formed perovskite active layer due to the continuous deprotonation of the FAI by the basic $\mathrm{ZnO}$ surface. a
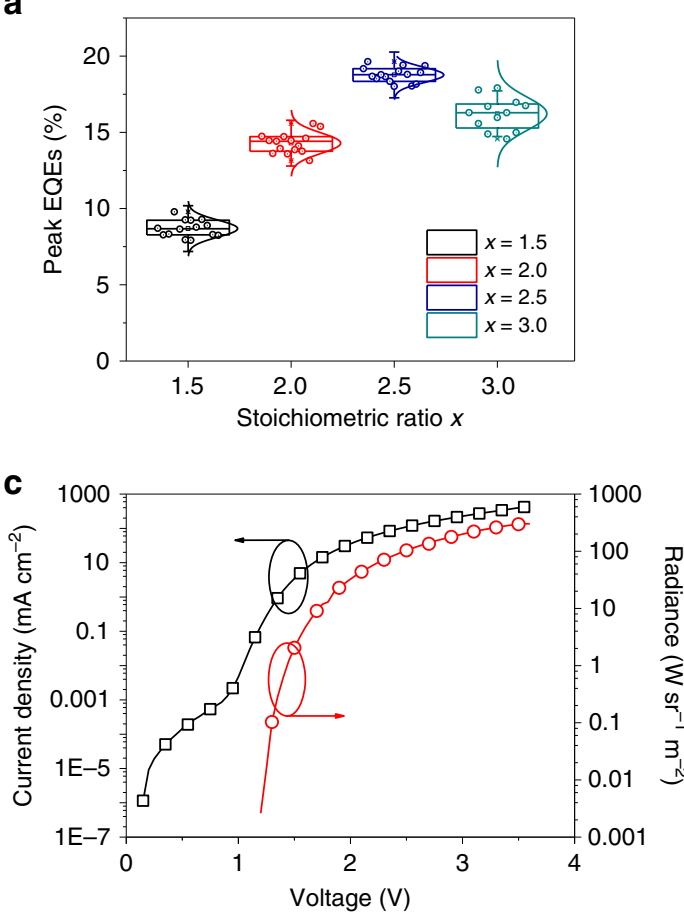

b

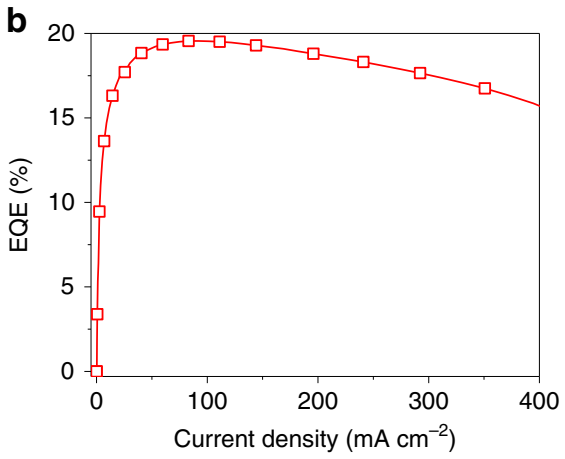

d

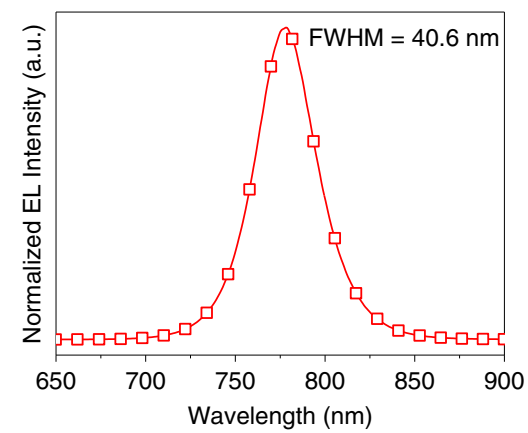

Fig. 5 Device performance of perovskite LEDs with TTDDA passivation. a Statistical distributions of peak EQEs of perovskite LEDs fabricated from TTDDAcontaining precursors with different component ratios. b, $\mathbf{c} E Q E-J(\mathbf{b}), J \& R-V(\mathbf{c})$ curves of the champion device with TTDDA passivation. $\mathbf{d}$ Normalized EL spectrum of the champion device operated at an applied voltage of $3.5 \mathrm{~V}$. For all box plots, the line in the center is the median value. The interquartile range (IQR) represents 25th-75th percentile of the EQE values. Whiskers show the extent of the whole EQE values, extending to 1.5 IQR in box-plots 
Perovskite films and LEDs with further defect passivation. Having revealed the critical role of the perovskite precursor stoichiometry and the unique interfacial reactions at the $\mathrm{ZnO} /$ perovskite interfaces, it is natural for us to investigate whether our finding is compatible with state-of-the-art molecular passivation that has boosted the device performance. A diamine passivation molecule, i.e., 4,7,10-trioxa-1,13-tridecanediamine (TTDDA), which was recently proven efficient in reducing the defect states in the perovskite films, is incorporated in the precursor solutions $^{38}$. In the FTIR spectra of the resulting perovskite films, we observe a distinct C-H stretching $(v \mathrm{C}-\mathrm{H})$ at $2865 \mathrm{~cm}^{-1}$ from the TTDDA (Supplementary Figure 9), which exists in neither the neat FAI film nor the pristine perovskite film, confirming the existence of TTDDA molecules in the resulting perovskite films. As a result of the TTDDA passivation, the non-radiative recombination in perovskite film is further suppressed, as indicated by improved PLQE values up to around $70 \%$ and prolonged charge carrier lifetime (Supplementary Figure 10a, b).

We notice that the incorporation of TTDDA results in neither low-dimensional phases nor impurities (Supplementary Figure $10 \mathrm{c}, \mathrm{d})$. With the same film processing conditions, we obtain highly luminescent perovskite films on the $\mathrm{ZnO} / \mathrm{PEIE}$ substrate while the formation of $\alpha$-phase perovskite on $\mathrm{SnO}_{2} / \mathrm{PEIE}$ and $\mathrm{TiO}_{\mathrm{x}} / \mathrm{PEIE}$ substrates is still hindered as evidenced by the absorption and PL results (Supplementary Figure 11). These results further demonstrate the critical role of the basic $\mathrm{ZnO} / \mathrm{PEIE}$ substrate on promoting the perovskite crystallization during the film formation process.

We fabricate perovskite LEDs with the TTDDA passivation and show the device results in Fig. 5a and Supplementary Figure 12. Consistent with the devices without TTDDA, we observe the same trend in the device efficiency, with the $x=2.5$ devices giving the highest performance. The champion device with TTDDA passivation shows a peak EQE of $19.6 \%$ along with a high radiance of $301.8 \mathrm{~W} \mathrm{sr}^{-1} \mathrm{~m}^{-2}$ (Fig. 5b, c). In addition, the typical electroluminescence (EL) spectrum of the champion device shows a narrow emission at around $780 \mathrm{~nm}$ with a fullwidth at half-maximum (FWHM) of around $40 \mathrm{~nm}$, which represents one of the narrowest EL emission spectra in NIR region (Fig. 5d) ${ }^{6,21}$. We further characterize the device operational stability, which is very important for practical applications of LEDs (Supplementary Figure 13). We find that the device based on the TTDDA-passivated perovskite film achieves a $T_{50}$ lifetime (the time for device to decay to $50 \%$ of its peak $\mathrm{EQE}$ value) of $7 \mathrm{~h}$ under a constant current density of $20 \mathrm{~mA} \mathrm{~cm}^{-2}$.

\section{Discussion}

The gathered information on the synergistic effects of the precursor stoichiometry and the deprotonation of the organic cations by the basic $\mathrm{ZnO}$ agrees well with previous efficient LEDs based on methylammonium (MA), FA or hybrid multiple-quantumwells (MQWs) structure perovskites, especially those with $\mathrm{ZnO} /$ PEIE as the bottom electron transport layer ${ }^{1,6,26,34}$. In order to demonstrate the generality of the deprotonation on promoting the perovskite film growth, we prepare MA-based perovskite films (MAI:PbI $\left.{ }_{2}=2.5\right)$ on the three different substrates. We observe typical UV-vis absorption and high crystalline feature of purephase $\mathrm{MAPbI}_{3}$ on the $\mathrm{ZnO} / \mathrm{PEIE}$ substrate (Supplementary Figure 14). Similar to the $\mathrm{FAPbI}_{3}$, the $\mathrm{MAPbI}_{3}$ perovskite films deposited on $\mathrm{TiO}_{x} / \mathrm{PEIE}$ and $\mathrm{SnO}_{2} /$ PEIE exhibit optical properties of the intermediate states, with no crystalline perovskites formed after the thermal annealing.

We believe that the results in this study are general for the fabrication of the $2 \mathrm{D}, 3 \mathrm{D}$ or quasi-2D perovskite films that contain organic cations, which can be deprotonated by the basic
$\mathrm{ZnO}$ surface during the processing conditions. In previous studies, the enhanced device performance of perovskite LEDs fabricated on $\mathrm{ZnO} / \mathrm{PEIE}$ was ascribed to improved surface wettability or reduced work function of the substrates ${ }^{40}$. However, in light of our findings in the present study, we believe that the precursor stoichiometry and the unique interfacial reactions during the perovskite crystallization induced by basic $\mathrm{ZnO}$ play very important roles in achieving high device performance.

It has been demonstrated that a continuous deprotonation reaction of the perovskite film by $\mathrm{ZnO}$ is harmful to the film and device stability, especially under an elevated temperature during the device operation. To further demonstrate the effect of interfacial interactions on the stability, we carry out in-situ PL measurements of the perovskite film on $\mathrm{ZnO} / \mathrm{PEIE}$. A recent report revealed that the temperature of a working perovskite LED based on mixed-cation perovskite of $\left(\mathrm{Cs}_{0.2} \mathrm{FA}_{0.8}\right) \mathrm{PbI}_{2.8} \mathrm{Br}_{0.2}$ could reach $41^{\circ} \mathrm{C}$ under a constant current density of $40 \mathrm{~mA} \mathrm{~cm}{ }^{-2}$, ref. ${ }^{41}$. We measure the evolution of the PL emission from the perovskite film at $60^{\circ} \mathrm{C}$, which represents a reasonably high temperature during device operation. We observe no change of the PL emission peak and slight increase of the PL intensity after $17 \mathrm{~h}$ heating (Supplementary Figure 15), suggesting that the interfacial reactions between the $\mathrm{ZnO} / \mathrm{PEIE}$ and the perovskite active layer are negligible under this mild temperature. The degradation mechanisms and critical factors affecting the device stability require further investigations.

In summary, we have demonstrated the critical role of the synergistic effect of the precursor stoichiometry and the interfacial reaction on achieving high-efficiency perovskite LEDs. We have observed efficient passivation of the excess organic halide on the defects in perovskite films. More importantly, deprotonation of these excess organic cations by a buried surface with a higher IEP value is beneficial to promote the transition of the intermediate phases into high-luminescence perovskite layers. In addition, the deprotonation process is compatible with state-ofthe-art passivation molecules. We believe that the key findings in this work provide insights for further development of highperformance perovskite LEDs, and offer inspirations for controllable deposition of the perovskite films for other optoelectronic devices on substrates with different surface properties.

\section{Methods}

Perovskite precursor solutions. The perovskite precursor solutions were prepared by mixing FAI, CsI and $\mathrm{PbI}_{2}$ with a molar ratio of $x$ in $\mathrm{N}, \mathrm{N}$ Dimethylformamide (DMF, anhydrous) at a concentration of $0.115 \mathrm{M}\left(\mathrm{PbI}_{2}\right)$ $\left((0.1 \mathrm{CsI}+0.9 \mathrm{FAI}): \mathrm{PbI}_{2}=x, x=1.0-3.0\right)$. For the precursor with TTDDA passivation molecules, $17.2 \mathrm{mM}$ TTDDA was added into the solution. MA-based perovskite precursor solution with a concentration of $0.15 \mathrm{M}$ was prepared by dissolving MAI and $\mathrm{PbI}_{2}$ with a molar ratio of 2.5:1 in DMF. All the prepared precursor solutions were stirred at $60^{\circ} \mathrm{C}$ for over $2 \mathrm{~h}$ before the film preparation.

Substrates preparation. ITO coated glass substrates were washed in the detergent aqueous solution prior to $10 \mathrm{~min}$ UV-ozone treatment. Tin (IV) oxide $\left(\mathrm{SnO}_{2}\right)$ colloidal solution and titanium oxide $\left(\mathrm{TiO}_{x}\right)$ precursor solution were prepared according to previous literatures ${ }^{42,43}$. Typically, the $\mathrm{SnO}_{2}$ colloidal solution was purchased from Alfa Aesar ( $15 \mathrm{wt} \%$ in water colloidal dispersion) and was diluted to $3 \mathrm{wt} \%$ with deionized water for film deposition. $\mathrm{TiO}_{\mathrm{x}}$ precursor solution was prepared by diluting the titanium iso-propoxide $(2 \mathrm{M}, 750 \mu \mathrm{L})$ in iso-propanol (IPA, $10 \mathrm{~mL}$ ) under vigorous stirring condition. After that, $70 \mu \mathrm{L} \mathrm{HCl}$ solution $(2$ $\mathrm{M})$ was added drop-wisely to the solution. The solution was further stirred for $12 \mathrm{~h}$ before use. $\mathrm{ZnO}$ nanoparticles were synthesised by a solution precipitation method $^{44}$. Typically, $1.5 \mathrm{mmol}$ zinc acetate hydrate $\left(\mathrm{Zn}(\mathrm{Ac})_{2} \cdot 2 \mathrm{H}_{2} \mathrm{O}\right)$ in $15 \mathrm{~mL}$ dimethyl sulfoxide (DMSO) was stirred at $30^{\circ} \mathrm{C}$. Then $2.8 \mathrm{mmol}$ tetramethylammonium hydroxide pentahydrate (TMAH $\left.5 \mathrm{H}_{2} \mathrm{O}\right)$ in $5 \mathrm{~mL}$ ethanol was dropped in $\mathrm{Zn}(\mathrm{Ac})_{2} \cdot 2 \mathrm{H}_{2} \mathrm{O}$ solution. The solution was kept stirring for $24 \mathrm{~h}$ prior to the precipitation process. The $\mathrm{ZnO}$ nanoparticles were precipitated by ethyl acetate, and washed with ethanol and ethyl acetate for one more time. Finally, the obtained $\mathrm{ZnO}$ nanoparticles were dispersed in ethanol $(8 \mathrm{~mL})$ and filtered with a $0.45 \mu \mathrm{m}$ PTFE filter before use. The PEIE dispersion was diluted in IPA solution with a concentration of $0.05 \mathrm{wt} \%$. The metal oxide films were prepared by spin-coating the solution on ITO substrates in ambient conditions. For $\mathrm{SnO}_{2}$ and $\mathrm{TiO}_{\mathrm{x}}$ 
substrates, the films were annealed at $150^{\circ} \mathrm{C}$ for 30 and $90 \mathrm{~min}$, respectively. PEIE was spin-coated on top of the metal oxide films at 5000 r.p.m. for $30 \mathrm{~s}$ and then annealed at $100^{\circ} \mathrm{C}$ for $10 \mathrm{mins}$ in the glove-box.

Device fabrication and characterization. Perovskite LEDs in this work were fabricated in a nitrogen filled glove-box. The perovskite films were spin-coated at 3000 r.p.m. for $30 \mathrm{~s}$ and then annealed on a pre-heated hotplate at $100^{\circ} \mathrm{C}$ for 10 min. TFB (12 mg ml-1 in chlorobenzene) layer was spin-coated on top of the perovskite film at 3000 r.p.m. for $30 \mathrm{~s}$. After that, $7 \mathrm{~nm} \mathrm{MoO}$ and $100 \mathrm{~nm} \mathrm{Ag}$ were deposited as the electrode in the thermal evaporator. The device active area is $7.25 \mathrm{~mm}^{2}$. The fabricated perovskite LEDs were measured in a glove-box at room temperature. A Keithley 2400 source meter was used to collect the current density and the driving voltage. An integrating sphere together with a QE Pro spectrometer (Ocean Optics) were used to collect the light emission. The applied voltage started from $0 \mathrm{~V}$ and increased with a step of $0.05 \mathrm{~V}$, lasting for $300 \mathrm{~ms}$ at each voltage step for stabilisation and measurements. The integrating sphere-spectrometer system was calibrated by a Vis-NIR radiometric calibration sources for absolute light intensity and a HG-1 calibration source for wavelength (Ocean Optics). The operational lifetime measurements of the LEDs were conducted using the same setup under a constant current density of $20 \mathrm{~mA} \mathrm{~cm}^{-2}$. The layout of the setup for LED measurements is displayed in Supplementary Figure 16.

Precursor and perovskite film characterizations. Size distributions of the colloids in perovskite precursor were measured by a zeta potential analyser (Brookhaven ZetaPlus). Morphology images of the perovskite films were obtained by a FEI (Quanta $200 \mathrm{FEG}$ ) scanning electron microscopy under a voltage of $5 \mathrm{kV}$. $\mathrm{X}$-ray diffraction (XRD) patterns were collected using an X-ray diffractometer (Panalytical X'Pert Pro) with $\mathrm{CuK} \alpha$ radiation. The X-ray was generated on a Copper target and the wavelength of the X-ray was $1.54 \AA$.

Ultraviolet-visible (UV-Vis) absorbance spectra were obtained with a PerkinElmer Lambda 900 in transmission mode. Steady state PL spectra were obtained with a $450 \mathrm{~nm}$ excitation laser and an Andor spectrometer (Shamrock sr303i-B, coupled to a Newton EMCCD Si array detector). The intensity-dependent PLQE results of perovskite films were obtained by an integrated system of a $450 \mathrm{~nm}$ continuous wavelength laser, optical fiber, spectrometer and integrating sphere. Time-correlated Single Photon Counting (TCSPC) PL decays were obtained by using an Edinburgh Instruments spectrometer (FLS980) with a 633-nm pulsed laser (less than $100 \mathrm{ps}, 0.1 \mathrm{MHz}$, YSL Supercontinuum Source SC-PRO). The excitation laser intensity was set at $0.5 \mathrm{~mW} \mathrm{~cm}-2$ ). In-situ PL spectra of perovskite films were obtained with a QE Pro spectrometer (Ocean Optics). The spectra were collected right after placing the samples on a pre-heated hotplate. A $450 \mathrm{~nm}$ LED laser was used to excite the film samples.

Fourier-transform infrared (FTIR) spectra of perovskite films were recorded by a PIKE MIRacle Vertex 70 Spectrometer (Bruker) using a DLaTGS detector at room temperature. The spectrometer is equipped with a PIKE MIRacle ${ }^{\mathrm{TM}}$ attenuated total reflectance (ATR) accessory as the sampling accessory. The measuring system was continuously purged with $\mathrm{N}_{2}$ before and during the measurements. The spectra were acquired at $2 \mathrm{~cm}^{-1}$ resolution with 30 scans over a wavenumber range between 4000 and $1000 \mathrm{~cm}^{-1}$. As for the perovskite films on different substrates, background spectra were obtained by measuring $\mathrm{SnO}_{2} / \mathrm{PEIE}$, $\mathrm{TiO}_{\mathrm{x}} / \mathrm{PEIE}$ and $\mathrm{ZnO} / \mathrm{PEIE}$ substrates. The presented spectra were baselinedcorrected by subtracting a linear baseline over the spectral ranges.

\section{Data availability}

The datasets generated and/or analysed during the current study are available from the corresponding author on reasonable request.

Received: 11 February 2019 Accepted: 22 May 2019

Published online: 27 June 2019

\section{References}

1. Cao, Y. et al. Perovskite light-emitting diodes based on spontaneously formed submicrometre-scale structures. Nature 562, 249-253 (2018).

2. Kumar, S. et al. Ultrapure green light-emitting diodes using two-dimensional formamidinium perovskites: achieving recommendation 2020 color coordinates. Nano Lett. 17, 5277-5284 (2017).

3. Protesescu, L. et al. Nanocrystals of cesium lead halide perovskites $\left(\mathrm{CsPbX}_{3}\right.$, $\mathrm{X}=\mathrm{Cl}, \mathrm{Br}$, and I): novel optoelectronic materials showing bright emission with wide color gamut. Nano Lett. 15, 3692-3696 (2015).

4. Chiba, T. et al. Anion-exchange red perovskite quantum dots with ammonium iodine salts for highly efficient light-emitting devices. Nat. Photon 12, 681-687 (2018).

5. Dong, Q. et al. Electron-hole diffusion lengths $>175 \mu \mathrm{m}$ in solution-grown $\mathrm{CH}_{3} \mathrm{NH}_{3} \mathrm{PbI}_{3}$ single crystals. Science 347, 967-970 (2015).
6. Wang, N. et al. Perovskite light-emitting diodes based on solution-processed self-organized multiple quantum wells. Nat. Photon 10, 699-704 (2016).

7. Zhao, B. et al. High-efficiency perovskite-polymer bulk heterostructure lightemitting diodes. Nat. Photon 12, 783-789 (2018).

8. Lin, K. et al. Perovskite light-emitting diodes with external quantum efficiency exceeding 20 per cent. Nature 562, 245-248 (2018).

9. Song, J. et al. Quantum dot light-emitting diodes based on inorganic perovskite cesium lead halides $\left(\mathrm{CsPbX}_{3}\right)$. Adv. Mater. 27, 7162-7167 (2015).

10. Wang, H. et al. Trifluoroacetate induced small-grained $\mathrm{CsPbBr}_{3}$ perovskite films result in efficient and stable light-emitting devices. Nat. Commun. 10, 665 (2019).

11. Xiao, Z. et al. Efficient perovskite light-emitting diodes featuring nanometresized crystallites. Nat. Photon 11, 108-115 (2017).

12. Cho, H. et al. Overcoming the electroluminescence efficiency limitations of perovskite light-emitting diodes. Science 350, 1222-1225 (2015).

13. Lee, J. W. et al. In-situ formed type I nanocrystalline perovskite film for highly efficient light-emitting diode. ACS Nano 11, 3311-3319 (2017).

14. Prakasam, V. et al. Efficient perovskite light-emitting diodes: effect of composition, morphology, and transport layers. ACS Appl. Mater. Interfaces 10, 41586-41591 (2018)

15. $\mathrm{Bi}, \mathrm{C}$. et al. Non-wetting surface-driven high-aspect-ratio crystalline grain growth for efficient hybrid perovskite solar cells. Nat. Commun. 6, 7747 (2015).

16. Wu, Z. et al. Efficient planar heterojunction perovskite solar cells employing graphene oxide as hole conductor. Nanoscale 6, 10505-10510 (2014).

17. Wojciechowski, K. et al. Heterojunction modification for highly efficient organic-inorganic perovskite solar cells. ACS Nano 8, 12701-12709 (2014).

18. Zhang, $\mathrm{H}$. et al. Pinhole-free and surface-nanostructured $\mathrm{NiO}_{\mathrm{x}}$ film by roomtemperature solution process for high-performance flexible perovskite solar cells with good stability and reproducibility. ACS Nano 10, 1503-1511 (2016)

19. Yang, J. et al. Origin of the thermal instability in $\mathrm{CH}_{3} \mathrm{NH}_{3} \mathrm{PbI}_{3}$ thin films deposited on ZnO. Chem. Mater. 27, 4229-4236 (2015).

20. Tan, Z. K. et al. Bright light-emitting diodes based on organometal halide perovskite. Nat. Nanotech 9, 687-692 (2014).

21. Yuan, M. et al. Perovskite energy funnels for efficient light-emitting diodes. Nat. Nanotech 11, 872-877 (2016).

22. Yang, X. et al. Efficient green light-emitting diodes based on quasi-twodimensional composition and phase engineered perovskite with surface passivation. Nat. Commun. 9, 570 (2018).

23. $\mathrm{Li}, \mathrm{Z}$. et al. Stabilizing perovskite structures by tuning tolerance factor: formation of formamidinium and cesium lead iodide solid-state alloys. Chem. Mater. 28, 284-292 (2016).

24. Lee, J. W. et al. Formamidinium and cesium hybridization for photo-and moisture-stable perovskite solar cell. Adv. Energy Mater. 5, 1501310 (2015).

25. McMeekin, D. P. et al. A mixed-cation lead mixed-halide perovskite absorber for tandem solar cells. Science 351, 151-155 (2016).

26. Zou, W. et al. Minimising efficiency roll-off in high-brightness perovskite light-emitting diodes. Nat. Commun. 9, 608 (2018).

27. Ma, F. et al. Stable $\alpha / \delta$ phase junction of formamidinium lead iodide perovskites for enhanced near-infrared emission. Chem. Sci. 8, 800-805 (2017).

28. Liu, P. et al. Halide-rich synthesized cesium lead bromide perovskite nanocrystals for light-emitting diodes with improved performance. Chem. Mater. 29, 5168-5173 (2017).

29. Baena, J. P. C. et al. Highly efficient planar perovskite solar cells through band alignment engineering. Energy Environ. Sci. 8, 2928-2934 (2015).

30. Heo, J. H. et al. Planar $\mathrm{CH}_{3} \mathrm{NH}_{3} \mathrm{PbI}_{3}$ perovskite solar cells with constant $17.2 \%$ average power conversion efficiency irrespective of the scan rate. Adv. Mater. 27, 3424-3430 (2015).

31. Song, Z. et al. Impact of processing temperature and composition on the formation of methylammonium lead iodide perovskites. Chem. Mater. 27, 4612-4619 (2015).

32. Zhao, B. et al. Thermodynamically stable orthorhombic $\gamma-\mathrm{CsPbI}_{3}$ thin films for high-performance photovoltaics. J. Am. Chem. Soc. 140, 11716-11725 (2018).

33. Parks, G. A. The isoelectric points of solid oxides, solid hydroxides, and aqueous hydroxo complex systems. Chem. Rev. 65, 177-198 (1965).

34. Yuan, Z., Bai, S., Yan, Z., Liu, J. M. \& Gao, F. Room-temperature film formation of metal halide perovskites on n-type metal oxides: the catalysis of $\mathrm{ZnO}$ on perovskite crystallization. Chem. Commun. 54, 6887-6890 (2018).

35. Zhao, Y. et al. Sequential multi-drop coating method for large crystallized $\alpha$ $\left(\mathrm{NH}_{2}\right)_{2} \mathrm{CHPbI}_{3}$ and mixed-organic-cation perovskite films for highly efficient mesoscopic perovskite solar cells. J. Power Sources 359, 147-156 (2017).

36. Petrov, A. A. et al. Formamidinium iodide: crystal structure and phase transitions. Acta Cryst. E 73, 569-572 (2017).

37. Lee, J. H., Bristowe, N. C., Bristowe, P. D. \& Cheetham, A. K. Role of hydrogen-bonding and its interplay with octahedral tilting in $\mathrm{CH}_{3} \mathrm{NH}_{3} \mathrm{PbI}_{3}$. Chem. Commun. 51, 6434-6437 (2015) 
38. $\mathrm{Xu}, \mathrm{W}$. et al. Rational molecular passivation for high-performance perovskite light-emitting diodes. Nat. Photon. 13, 418-424 (2019).

39. Choi, K. et al. Thermally stable, planar hybrid perovskite solar cells with high efficiency. Energy Environ. Sci. 11, 3238-3247 (2018).

40. Wang, J. et al. Interfacial control toward efficient and low-voltage perovskite light-emitting diodes. Adv. Mater. 27, 2311-2316 (2015).

41. Zhao, L., Lee, K. M., Roh, K., Khan, S. U. Z. \& Rand, B. P. Improved outcoupling efficiency and stability of perovskite light-emitting diodes using thin emitting layers. Adv. Mater. 31, e1805836 (2019).

42. Docampo, P., Ball, J. M., Darwich, M., Eperon, G. E. \& Snaith, H. J. Efficient organometal trihalide perovskite planar-heterojunction solar cells on flexible polymer substrates. Nat. Commun. 4, 2761 (2013).

43. Jiang, Q. et al. Enhanced electron extraction using $\mathrm{SnO}_{2}$ for high-efficiency planar-structure $\mathrm{HC}\left(\mathrm{NH}_{2}\right)_{2} \mathrm{PbI}_{3}$-based perovskite solar cells. Nat. Energy 2, 16177 (2016).

44. Bai, S. et al. High-performance planar heterojunction perovskite solar cells: preserving long charge carrier diffusion lengths and interfacial engineering. Nano Res. 7, 1749-1758 (2014).

\section{Acknowledgements}

We thank Mr. Fusheng Ma and Prof. Juan Qiao for the DLS measurements and analysis. We thank Dr. Xianjie Liu for XPS measurement and analysis. We thank Dr. Xiaoke Liu, Dr. Yuqing Huang and Dr. Feng Wang for helpful discussions. This work was supported by the ERC Starting Grant (717026), the European Commission Marie Skłodowska-Curie Actions (691210), the National Key Research and Development Program of China (2016YFA0202402), Jiangsu High Educational Natural Science Foundation (18KJA430012), the Priority Academic Program Development of Jiangsu Higher Education Institutions, the 111 program and Collaborative Innovation Center of Suzhou Nano Science and Technology (NANO-CIC). Z.Y. would like to thank the financial support from the China Scholarship Council. W.X. thanks the National Natural Science Foundation of China (61704077), the Natural Science Foundation of Jiangsu Province (BK20171007). W.X. is a Wenner-Gren Postdoc Fellow. F.G. is a Wallenberg Academy Fellow.

\section{Author contributions}

F.G. and S.B. supervised the work. Z.Y. carried out the perovskite LED devices fabrication and measurements, films characterizations. W.X contributed to the device optimization and C.K. conducted device stability test. Y.M., P.K., P.L. and J.L. conducted the TCSPC and PLQE measurements and analysed the data under the supervision of J. W. The FTIR measurements were carried out and analysed with the assistance of Z.H., S.B. and Z.Y. wrote the manuscript, and B.S., J.W. and F.G. provided revisions. All authors discussed the results and commented on the manuscript.

\section{Additional information}

Supplementary Information accompanies this paper at https://doi.org/10.1038/s41467019-10612-3.

Competing interests: The authors declare no competing interests.

Reprints and permission information is available online at http://npg.nature.com/ reprintsandpermissions/

Peer review information: Nature Communications thanks the anonymous reviewer(s) for their contribution to the peer review of this work.

Publisher's note: Springer Nature remains neutral with regard to jurisdictional claims in published maps and institutional affiliations.

(c) (i) Open Access This article is licensed under a Creative Commons Attribution 4.0 International License, which permits use, sharing, adaptation, distribution and reproduction in any medium or format, as long as you give appropriate credit to the original author(s) and the source, provide a link to the Creative Commons license, and indicate if changes were made. The images or other third party material in this article are included in the article's Creative Commons license, unless indicated otherwise in a credit line to the material. If material is not included in the article's Creative Commons license and your intended use is not permitted by statutory regulation or exceeds the permitted use, you will need to obtain permission directly from the copyright holder. To view a copy of this license, visit http://creativecommons.org/ licenses/by/4.0/.

(C) The Author(s) 2019 